\title{
Evaluation of the relationship between KIM-1 and suPAR level in COVID-19 patients and a different perspective on suPAR
}

\author{
Buğra Kerget ${ }^{1}$, Ferhan Kerget ${ }^{2}$, Alperen Aksakal ${ }^{2}$, Seda Aşkın², Elif Yilmazel Ucar ${ }^{2}$, and \\ Leyla Sağlam ${ }^{1}$ \\ ${ }^{1}$ Ataturk Universitesi \\ ${ }^{2}$ Affiliation not available
}

February 15, 2021

\begin{abstract}
Objective: COVID-19 is one of the most important health problems concerning the last century and our knowledge of the disease is still limited. In our study, we aimed to compare serum-soluble urokinase plasminogen activator receptor (suPAR) and kidney injury molecule-1 (KIM-1) level with clinical course in COVID-19 patients. Methods: Our study included 102 patients over the age of 18 who were diagnosed with Covid-19 between September 2020 and December 2020 by taking nasopharyngeal swap and using real time PCR method and 30 volunteer medical personnel over the age of 18 who were PCR negative after the nasopharyngeal swap. KIM-1 and suPAR were measured by enzyme-linked immunosorbent assay. Results: NLR, LDH, prothrombin time, CRP, $\mathrm{PaO} 2 / \mathrm{FiO} 2$, D-Dimer, ferritin and fibrinogen levels, which have been mentioned in previous studies to be of prognostic importance for COVID-19, were observed to be higher in the severely ill group ( $p=0,001,0,001,0,05$, 0,001, 0,001, 0,005, 0,001, 0,001 respectively). suPAR and KIM-1 levels were statistically significantly higher in patient groups compared to the control group ( $\mathrm{p}=0.001$ for all). While suPAR level was statistically significantly lower in severe patients compared to moderate patients $(\mathrm{p}=0.034)$, KIM-1 level was observed to be higher in severe patients $(\mathrm{p}=0.001)$. Conclusion: The increased level of KIM-1 in severe patients, which is thought to play an important role in the endocytosis of SARS-CoV-2 to the cell, may have an important place for the therapeutic target in the future. SuPAR can be considered to play an important role especially in the defense mechanism and fibrinolysis and its decreased level in severe patients may be associated with poor prognosis in the early period. However, extensive studies are needed to reach a definitive opinion about suPAR.
\end{abstract}

\section{Evaluation of the relationship between KIM-1 and suPAR level in COVID-19 patients and a different perspective on suPAR}

Short title: KIM-1 and suPAR level in COVID-19

Buğra Kerget ${ }^{1}$, Ferhan Kerget ${ }^{2}$, Alperen Aksakal ${ }^{3}$, Seda Aşkın ${ }^{4}$, Elif Yılmazel Uçar ${ }^{1}$, Leyla Sağlam ${ }^{1}$

${ }^{1}$ Depertment of Pulmonary Diseases, Ataturk University School of Medicine, Yakutiye, Erzurum, Turkey

${ }^{2}$ Depertmant of Infection Diseases and Clinical Microbiology, Health Sciences University Erzurum Regional Education and Research Hospital, Yakutiye, Erzurum, Turkey

${ }^{3}$ Depertmant of Pulmonary Diseases, Health Sciences University Erzurum Regional Education and Research Hospital, Yakutiye, Erzurum, Turkey

${ }^{4}$ Depertmant of Biochemistry, Ataturk University School of Medicine, Yakutiye, Erzurum, Turkey

All authors disclosure no Conflict of Interest.

*Corresponding Author: 
Buğra Kerget,MD

Yakutiye Medical Research Center

Chest Disease Department

25240, Yakutiye

Erzurum, TURKEY

Tel: +904423447446

Fax: +904423446528

bjkerget1903@gmail.com

Evaluation of the relationship between KIM-1 and suPAR level in COVID-19 patients and a different perspective on suPAR

\begin{abstract}
Objective: COVID-19 is one of the most important health problems concerning the last century and our knowledge of the disease is still limited. In our study, we aimed to compare serum-soluble urokinase plasminogen activator receptor (suPAR) and kidney injury molecule-1 (KIM-1) level with clinical course in COVID-19 patients.

Methods: Our study included 102 patients over the age of 18 who were diagnosed with Covid-19 between September 2020 and December 2020 by taking nasopharyngeal swap and using real time PCR method and 30 volunteer medical personnel over the age of 18 who were PCR negative after the nasopharyngeal swap. KIM-1 and suPAR were measured by enzyme-linked immunosorbent assay.

Results: NLR, LDH, prothrombin time, CRP, $\mathrm{PaO}_{2} / \mathrm{FiO}_{2}$, D-Dimer, ferritin and fibrinogen levels, which have been mentioned in previous studies to be of prognostic importance for COVID-19, were observed to be higher in the severely ill group ( $\mathrm{p}=0,001,0,001,0,05,0,001,0,001,0,005,0,001,0,001$ respectively). suPAR and KIM-1 levels were statistically significantly higher in patient groups compared to the control group ( $\mathrm{p}=0.001$ for all). While suPAR level was statistically significantly lower in severe patients compared to moderate patients $(\mathrm{p}=0.034)$, KIM-1 level was observed to be higher in severe patients $(\mathrm{p}=0.001)$.
\end{abstract}

Conclusion: The increased level of KIM-1 in severe patients, which is thought to play an important role in the endocytosis of SARS-CoV-2 to the cell, may have an important place for the therapeutic target in the future. SuPAR can be considered to play an important role especially in the defense mechanism and fibrinolysis and its decreased level in severe patients may be associated with poor prognosis in the early period. However, extensive studies are needed to reach a definitive opinion about suPAR.

Keywords: COVID-19, KIM-1, suPAR

\title{
What's already known about this topic?
}

The KIM-1 molecule, which was previously associated with acute kidney damage, is synthesized in proximal tubules after kidney damage and plays an important role in inflammation and immune response. Research has shown that the KIM-1 molecule, which is significantly synthesized in the lung and kidney, can be a receptor that facilitates the binding of SARS-CoV-2 to the cell surface through the IgV field and have put forward that it may be the cause of acute kidney damage in patients with Covid-19. Soluble urokinase plasminogen activator (suPAR), the dissolved form of urokinase plasminogen activator, has been another molecule thought to be responsible for acute kidney damage that may develop in Covid-19. However, suPAR may also play a role in fetal complications that may occur in the lungs and heart. suPAR plays an important role in the remodeling of tissues under inflammation and immune cell migration and activation.

What does this article add? 
KIM-1/TIM-1 acts as an important gateway to the entry of the virus into the cell and its increased level of severely ill patients seems to confirm this. Treatments for this gateway can play an important role in treatment in COVID-19. In addition, the falling level of suPAR, which plays a role in body defense and fibrinolisis, with the weight of the disease may be the cause of parenchymal fibrosis development in patients, increased predisposition to thromboembolic events and increased virulence. This issue seems to need to be confirmed through more extensive research.

\section{Introduction}

The pandemic has been a frequently used medical term for our world in the last year and whose meaning is known to everyone. Although pandemics have not left our world since the existence of humanity, the sociocultural, economic and psychological effects they create leave lasting damage to people. Covid-19 is the latest pandemic that our world has experienced, and while most affected people are prevalent with mild symptoms such as loss of taste and smell, sore throat, joint pain and headache, especially in individuals over 65 who have comorbidity, it can progress with morbidity and mortality comorbidity 1.

Acute respiratory failure syndrome has been one of the most important causes of mortality since the beginning of Covid-19 infection. With increased experience in patients, more frequent observance of thrombotic events and renal failure compared to known viral infections in followed patients has resulted in deepening of research $2,3$.

Initially, the angiotensin-converting enzyme 2 (ACE2) receptor was considered the most important receptor in the introduction of the virus into the cell and medical treatments were moving in this direction. However, later the discovery of the transmembrane protein serine protease-2 (TMPRSS2), which facilitates the introduction of the virus into the cell, and kidney injury molecule-1 (KIM-1), which is highly synthesized especially in the lung, and kidney, has caused the direction of treatment and target to change 4,5 .

The KIM-1 molecule, which was previously associated with acute kidney damage, is synthesized in proximal tubules after kidney damage and plays an important role in inflammation and immune response. Research has shown that the KIM-1 molecule, which is significantly synthesized in the lung and kidney, can be a receptor that facilitates the binding of SARS-CoV-2 to the cell surface through the IgV field and have put forward that it may be the cause of acute kidney damage in patients with Covid-19 5, 6 . Soluble urokinase plasminogen activator (suPAR), the dissolved form of urokinase plasminogen activator, has been another molecule thought to be responsible for acute kidney damage that may develop in Covid-19 7. However, suPAR may also play a role in fetal complications that may occur in the lungs and heart 8. suPAR plays an important role in the remodeling of tissues under inflammation and immune cell migration and activation. It also balances tissue and organ damage that may develop due to excessive inflammatory response 9 . Therefore, its importance increases even more, especially in a disease such as Covid-19, where the abnormal inflammatory response is involved. In our study, we aimed to investigate the role of serum suPAR and KIM-1 level in determining the relationship with clinical course in Covid-19 patients who did not develop acute renal failure in their follow-up.

\section{Material and methods}

Study design

As the standard, high-resolution computed tomography (HRCT) was performed for high-risk patients with COVID-19. In line with HRCT results, while patients who have observed opacity in bilateral frosted glass density showing peripheral placement in the foreground, sub segmental consolidation or linear opacities, paving stone view, inverse halo sign were evaluated as typical findings; patients with concordant clinical complaints, even if they had radiologically atypical findings, were hospitalized. After the hospitalization of the patients, hematological parameters, biochemical parameters to include liver and kidney function tests, clotting parameters, ferritin, D-Dimer, troponin-i, CRP, and arterial blood gas parameters were analysed. The current parameters of the patients were repeated daily.

Study group 
The 132 people included in our study were divided into 3 groups. Group 1; Asymptomatic volunteering health employees who came out negative as a result of PCR for Covid-19 (n:30), Group 2; Moderately ill patients with clinical signs of pneumonia with no signs of severe pneumonia (severe pneumonia: Pneumonia fitting any one of the following conditions: respiratory rate [?] 30 breaths/min; SpO2 [?] 92\%; patients with $>50 \%$ of lung infiltration rate) (n:62), Group 3: Severely ill patients who are admitted with severe pneumonia and develop macrophage activation syndrome in their follow-up (n:40).

\section{Exclusion Criteria}

Exclusion criteria were determined as the presence of chronic or clinically significant infectious or inflammatory conditions in the last month, asthma, chronic obstructive pulmonary disease (COPD), malignancy, invasive surgical intervention in the last month, uncontrolled hypertension, patients with high fasting blood sugar, diabetes, cerebrovascular disease, kidney disease and coronary artery disease. Anamnesis information and laboratory parameters taken during hospitalization were used in the evaluation of patients in terms of exclusion criteria. In terms of coronary artery disease, asthma, KOAH presence and diabetes, cardiology, chest diseases and internal medicine were consulted by clinics.

Definitions and Treatment

In patients, temperatures taken as axillary and found to be above $37.3 \mathrm{C}$ were defined as fever. In patients with high fever observed under treatment for Covid-19, blood, urine and culture of sputum for possible bacterial, fungal superinfections were taken and empirically given antibio therapies were revised according to culture results. Diagnosis and rating of acute respiratory failure was made according to Berlin 2015 diagnostic criteria ${ }^{10}$. If the cardiological specific troponin level of patients was observed above normal, it was evaluated in terms of cardiological pathologies that may have just developed with echocardiography. Coagulopathy was defined as 3 seconds above normal at the time of prothrombin and 5 seconds above normal at the level of partial thromboplastin. Treatment strategy according to the weight of the patients was made according to the Covid-19 adult diagnosis and treatment guide of the Ministry of Health of the Republic of Turkey. in case of symptoms such as resistant fever, which continues despite treatment, CRP and ferritin values, which are constantly high or continue to increase, cytopenias in the form of lymphopenia and thrombocytopenia, impaired liver function tests, pitofibrinogenemia or elevation in triglyceride values, patients were followed up for MAS D-dimer height. In case of increase in daily sequential measurements in these parameters and when this situation cannot be explained by secondary bacterial infections, $400 \mathrm{mg}$ of tosilizumab was applied to patients if there was no contraindication for MAS. After 24 hours, the appropriate clinical and laboratory response was not repeated in the patients received. However, the appropriate clinic and laboratory were repeated in the same dose in case of no response.

\section{Measurement of biochemical markers}

After 15 minutes of semi-supine rest, blood samples were obtained from an antecubital vein into tubes containing ethylenediaminetetraacetic acid (EDTA) to prevent coagulation. Troponin I concentrations were measured by chemiluminescent immunoassay using an Immulite 2500 (Siemens Medical Solutions, Erlangen, Germany). KIM-1 and suPAR were measured by enzyme-linked immunosorbent assay (Elabscience human ELISA kit, UK).

\section{Statistical analysis}

The data were analyzed using IBM SPSS Statistics for Windows version 20.0 (IBM Corp., Armonk, NY). Pearson's chi-square test and Mann-Whitney U test were used for intergroup comparisons of parametric data and nonnormally distributed numerical data, respectively. Independent-samples t test was used to compare demographic data and laboratory parameters between the groups. Wilcoxon analysis was used for intragroup comparisons of laboratory values during follow-up. Pearson correlation analysis was used to evaluate relationships between KIM-1 and suPAR levels and CRP, ferritin, D-dimer, lymphocyte, neutrophil/lymphocyte ratio, troponin-I, and $\mathrm{PaO}_{2} / \mathrm{FiO}_{2}$. A p-value less than 0.05 was considered statistically significant.

\section{Results}


The mean age of the 102 patients included in our study was $56.1 \pm 14.9$ percent. The mean age of the patients in the control group was $53.1 \pm 18.1$ percent. No statistically significant difference was observed between the patient and the control group $(\mathrm{p}=0.34) 59$ of the patients were male and 43 were female. The mean age of male patients was $56.3 \pm 15.8$ and female patients were $55.9 \pm 13.8$ percent. Statistical analysis of the mean age of patients by gender showed no significant difference between average age $(\mathrm{p}=0.9)$.

While we did not have patients who developed mortality during the follow-up, the need for mechanical ventilators in severe patients did not occur in the following process. Only 2 severe pneumonia patients developed a massive pulmonary thromboembolism setting in spite of the application of enoxaparin sodium at 12-hour intervals in accordance with weight.

Evaluation of laboratory parameters according to the disease severity belonging to COVID-19 patients included in our study was carried out in Table 1. NLR, LDH, prothrombin time, CRP, PaO2/FiO2, D-Dimer, ferritin and fibrinogen levels which have been mentioned in previous studies to have prognostic importance for COVID-19, were observed to be higher in the severely ill group ( $\mathrm{p}=0.001,0.001,0.05,0.001,0.001,0.005$, $0.001,0.001$ respectively). Comparison of the patient groups among themselves and the control group with suPAR and KIM-1 levels is shown in Table 2. Accordingly, suPAR and KIM-1 levels were statistically significantly higher to the control group in patient groups ( $\mathrm{p}=0.001$ for all). While suPAR level was statistically significantly lower in severe patients compared to middle patients $(\mathrm{p}=0.034)$, KIM-1 level was observed to be higher in severely ill patients $(\mathrm{p}=0.001)$.

Comparison of the suPAR and KIM-1 levels of patient groups among themselves and the control group is shown in Table 2. Accordingly, suPAR and KIM-1 levels were statistically significantly higher to the control group in patient groups $(\mathrm{p}=0.001$ for all). While suPAR level was statistically significantly lower in severe patients compared to middle patients $(\mathrm{p}=0.034)$, KIM-1 level was observed to be higher in severe patients $(\mathrm{p}=0.001)$.

While negative directional correlation was observed in the correlation analysis of suPAR level with age $(\mathrm{r}=-$ $0.197, \mathrm{p}=0.05)$, positive directional correlation was observed in correlation analysis with $\mathrm{PaO} 2 / \mathrm{FiO} 2$ level $(\mathrm{r}=0.316, \mathrm{p}=0.01)$ (Figure 1). Statistically significant difference was not observed in the correlational analysis of suPAR level with D-Dimer $(\mathrm{r}=-0,114, \mathrm{p}=0,255)$. While negative directional correlation was observed in the correlation analysis of KIM-1 level with $\mathrm{PaO} 2 / \mathrm{FiO} 2$ level (Figure 1), it was observed that it showed positive correlation with parameters associated with prognosis such as NLR and LDH ( $\mathrm{r}=-0,319, \mathrm{p}=0.01$, $\mathrm{r}=0.336, \mathrm{p}=0.01, \mathrm{r}=0.466, \mathrm{p}=0.01$ respectively).

\section{Discussion}

In our study, it was observed that laboratory parameters that have been observed to have prognostic importance in COVID-19 patients increased in correlation with the severity of the disease. In the evaluation of suPAR and KIM-1 levels, it was observed that suPAR level was low compared to moderate patients in severe patients, while KIM-1 level increased in line with the severity of the disease. In the correlation of $\mathrm{PaO} 2 / \mathrm{FiO} 2$ level with the parameters subject to our research, positive directional correlation was observed with suPAR level, while negative directional correlation was found with KIM-1 level.

SARS-CoV-2 is closely related to SARS-CoV and MERS-CoV, which are responsible for past outbreaks with significant morbidity and mortality. The rapid spread of COVID-19 has caused the pandemic and is now spreading globally. So far, the number of people infected due to COVID-19 is said to be over a hundred million, and the number is increasing day by day 11 .

Observing lymphopenia in most patients in the examination of laboratory parameters of COVID-19 patients suggested that $2019 \mathrm{nCoV}$ similar to SARS-CoV could have an effect on lymphocytes, especially T lymphocytes. Virus particles emanating from the respiratory mucosa and infecting other cells cause abnormal cytokine discharge in the body, which is called a cytokine storm. T lymphocyte damage is also important in cytokine storm formation ${ }^{12}$. After the cytokine storm, many proinflammatory cytokines, especially TNF-alpha, IL-1, IL-2, IL-6, nitric oxide, are released. These cytokines can cause increased vascular permeability, causing 
deterioration in tissue perfusion, as well as endothelial damage and microtombus formation. This increase in vascular permeability causes fluid accumulation in lung tissue and the interstitial area, resulting in an acute setting of respiratory failure. Positive results have been reported that IL- 1 and IL- 6 antagonists can be used to control this setting ${ }^{13,14}$.

The dense presence of the KIM-1 molecule in $\mathrm{T}$ cells gave it a different name: $\mathrm{T}$ cell/transmembrane, immunoglobulin, and mucin (TIM-1). The interaction of KIM-1/TIM-1 with T cells has played an important role in immune response, allergy, asthma, autoimmune diseases and response to viral infections ${ }^{5}$. In the preliminary evaluation of clinical interaction between KIM-1/TIM-1 and COVID-19 patients, the relationship with acute kidney damage was evaluated in the foreground ${ }^{15}$. Kim-1/TIM-1 has been observed to facilitate the entry of the virus into the cell, such as ACE-2 receptors via IgV. It has been observed that increased proinflammatory cytokine levels as a result of increased virulence may cause further progression of kidney damage ${ }^{6}$. In studies with SARS-CoV and MERS-CoV, it was also determined that the IgV unit provides ease of entry for other members of the family ${ }^{16}$. In another publication, which is also in the evaluation phase, it has been suggested that KIM-1/TIM-1 receptors are high in the lungs and kidneys and that the observed TW-37 molecule, which can inhibit anth-KIM-1/TIM-1 antibody and endocytosis, can be used as a therapeutic target ${ }^{5}$.

Humoral immunity plays an important role in controlling infection after the development of viremia. One of the chemotactic agents that plays an important role in the migration of these cells is uPAR. uPAR and its serum-dissolved form, suPAR, mediate the transformation of plasminogen into a plasmin.

By enabling a number of proteolytic activities to take place in the placenum extracellular matrix, it facilitates the migration of cells that play a role in the immune response ${ }^{8}$. Studies investigating the relationship of suPAR level with inflammatory diseases found that the level of diabetes mellitus, coronary artery disease, community origin, ventilator-related pneumonias, smoking, acute enflaming of chronic obstructive pulmonary disease and sepsis increased compared to the healthy control group $17,18,19,20,21,22,23,24,25$. It has also been shown that early mortality may have an important place in the pre-examination and survey in patients with sepsis. In the study evaluating patients with COVID-19, it was also assessed that there may be an early biomarker in patients who may require hospitalization in intensive care ${ }^{26}$.

In our study, it was observed that the laboratory parameters examined in hospitalization such as NLR, LDH, prothrombin time, CRP, $\mathrm{PaO} 2 / \mathrm{FiO} 2$, D-Dimer, ferritin and fibrinogen levels were higher in severe patients in line with previous studies conducted in patients with COVID-19. It was also observed that the KIM-1 level taken care of during hospitalization was high compared to moderately ill patients compared to severely ill patients. This can be assessed as the KIM-1, which mediates the endocytology of the virus in the cell, increases viremia.

In addition, increased viremia may have led to the development of MAS with abnormal cytokine discharge in these patients later on. In the evaluation of suPAR levels, we obtained a different observation with previous studies. Compared to the healthy control group, it was observed that the rising suPAR level in both moderate and severe COVID-19 patients was lower in severely ill patients than in moderate COVID-19 patients. SuPAR, which plays an important role in the migration of cells involved in body defense, can be evaluated as aggravating the disease due to the inability to create a low level adequate immune response in severe patients. In addition, the decreased level of this molecule, which plays a role in fibrinolytic activity, may have led to hypofibrinolytic activity, causing acute respiratory distress.

The most important limit observed in our study was that the number of our severely ill patients was small compared to the number of moderately ill patients. However, due to the effects of the level of parameters subject to our research on comorbidities, the exclusion of these patients caused our number to be small.

As a result, KIM-1/TIM-1 acts as an important gateway to the entry of the virus into the cell and its increased level of severely ill patients seems to confirm this. Treatments for this gateway can play an important role in treatment in COVID-19. In addition, the falling level of suPAR, which plays a role in body defense and fibrinolisis, with the weight of the disease may be the cause of parenchymal fibrosis development in patients, 
increased predisposition to thromboembolic events and increased virulence. This issue seems to need to be confirmed through more extensive research.

\section{References}

1. Yuki K, Fujiogi M, Koutsogiannaki S. COVID-19 pathophysiology: A review. Clinical immunology. 2020:108427.

2. Poyiadji N, Cormier P, Patel PY, et al. Acute pulmonary embolism and COVID-19. Radiology. 2020;297(3):E335-E338.

3. Kerget B, Akgun M, Dogan N. Atypical presentation of COVID-19: Acute renal failure. The Eurasian Journal of Medicine. 2020;52(2):224.

4. Asselta R, Paraboschi EM, Mantovani A, Duga S. ACE2 and TMPRSS2 variants and expression as candidates to sex and country differences in COVID-19 severity in Italy. 2020.

5. Ichimura T, Mori Y, Aschauer P, et al. KIM-1/TIM-1 is a Receptor for SARS-CoV-2 in Lung and Kidney. medRxiv. 2020.

6. Yang C, Zhang Y, Chen H, et al. Kidney injury molecule-1 is a potential receptor for SARS-CoV-2. bioRxiv. 2020.

7. Azam TU, Shadid HR, Blakely P, et al. Soluble Urokinase Receptor (SuPAR) in COVID-19-Related AKI. Journal of the American Society of Nephrology. 2020;31(11):2725-2735.

8. D'Alonzo D, De Fenza M, Pavone V. COVID-19 and pneumonia: a role for the uPA/uPAR system. Drug discovery today. 2020;25(8):1528-1534.

9. Hamie L, Daoud G, Nemer G, et al. SuPAR, an emerging biomarker in kidney and inflammatory diseases. Postgraduate medical journal.2018;94(1115):517-524.

10. Sjoding MW, Hofer TP, Co I, Courey A, Cooke CR, Iwashyna TJ. Interobserver reliability of the Berlin ARDS definition and strategies to improve the reliability of ARDS diagnosis. Chest.2018;153(2):361-367.

11. Peeri NC, Shrestha N, Rahman MS, et al. The SARS, MERS and novel coronavirus (COVID-19) epidemics, the newest and biggest global health threats: what lessons have we learned? International journal of epidemiology. 2020;49(3):717-726.

12. He R, Lu Z, Zhang L, et al. The clinical course and its correlated immune status in COVID-19 pneumonia. Journal of Clinical Virology. 2020;127:104361.

13. Kerget B, Kerget F, Koçak AO, et al. Are serum interleukin 6 and surfactant protein D levels associated with the clinical course of COVID-19? Lung. 2020;198(5):777-784.

14. Kerget B, Kerget F, Aksakal A, Aşkın S, Sağlam L, Akgün M. Evaluation of alpha defensin, IL-1 receptor antagonist, and IL-18 levels in COVID-19 patients with macrophage activation syndrome and acute respiratory distress syndrome. Journal of Medical Virology. 2020.

15. Luther T, Bulow-Anderberg S, Larsson A, et al. COVID-19 patients in intensive care develop predominantly oliguric acute kidney injury.Acta Anaesthesiologica Scandinavica. 2020.

16. Rabaan AA, Al-Ahmed SH, Haque S, et al. SARS-CoV-2, SARS-CoV, and MERS-COV: a comparative overview. Infez Med. 2020;28(2):174-184.

17. Wu C-Z, Chang L-C, Lin Y-F, et al. Urokinase plasminogen activator receptor and its soluble form in common biopsy-proven kidney diseases and in staging of diabetic nephropathy. Clinical biochemistry.2015;48(18):1324-1329.

18. Van Oort PM, Bos LD, Povoa P, et al. Soluble urokinase plasminogen activator receptor for the prediction of ventilator-associated pneumonia. ERJ open research. 2019;5(1). 
19. Tsai P-K, Tsao S-M, Yang W-E, Yeh C-B, Wang H-L, Yang S-F. Plasma soluble urokinase-type plasminogen activator receptor level as a predictor of the severity of community-acquired pneumonia.International journal of environmental research and public health. 2019;16(6):1035.

20. Gumus A, Altintas N, Cinarka H, et al. Soluble urokinase-type plasminogen activator receptor is a novel biomarker predicting acute exacerbation in COPD. International journal of chronic obstructive pulmonary disease. 2015;10:357.

21. Guthoff M, Wagner R, Randrianarisoa E, et al. Soluble urokinase receptor (suPAR) predicts microalbuminuria in patients at risk for type 2 diabetes mellitus. Scientific reports. 2017;7(1):1-7.

22. Theilade S, Lyngbaek S, Hansen T, et al. Soluble urokinase plasminogen activator receptor levels are elevated and associated with complications in patients with type 1 diabetes. Journal of internal medicine. 2015;277(3):362-371.

23. Eugen-Olsen J, Ladelund S, Sorensen LT. Plasma su PAR is lowered by smoking cessation: a randomized controlled study. European journal of clinical investigation. 2016;46(4):305-311.

24. Okulu E, Arsan S, Akin IM, et al. Serum levels of soluble urokinase plasminogen activator receptor in infants with late-onset sepsis. Journal of clinical laboratory analysis. 2015;29(5):347-352.

25. Hoenigl M, Raggam RB, Wagner J, et al. Diagnostic accuracy of soluble urokinase plasminogen activator receptor (suPAR) for prediction of bacteremia in patients with systemic inflammatory response syndrome. Clinical biochemistry. 2013;46(3):225-229.

26. Donadello K, Scolletta S, Covajes C, Vincent J-L. suPAR as a prognostic biomarker in sepsis. BMC medicine. 2012;10(1):1-9.

Figure legends

Figure 1. Correlation analysis of serum suPAR and KIM-1 levels of patients followed up due to COVID-19 done with $\mathrm{PaO} 2 / \mathrm{FiO} 2$

\section{Hosted file}

Table.ENG.pdf available at https://authorea.com/users/387698/articles/508958-evaluationof-the-relationship-between-kim-1-and-supar-level-in-covid-19-patients-and-a-differentperspective-on-supar

\section{Hosted file}

Figure 1.ENG.pdf available at https://authorea.com/users/387698/articles/508958-evaluationof-the-relationship-between-kim-1-and-supar-level-in-covid-19-patients-and-a-differentperspective-on-supar 